\title{
Mediaciones y pragmatismo 1
}

\author{
Mediations and pragmatism
}

\author{
Ornela Boix * y Pablo Semán ** \\ * Universidad Nacional de La Plata - Instituto de Investigaciones en Humanidades y \\ Ciencias Sociales - CONICET, Argentina | ornelaboix@gmail.com \\ ** Universidad Nacional de San Martín - Instituto de Altos Estudios Sociales - \\ CONICET, Argentina | pabloseman@hotmail.com
}

\section{PALABRAS CLAVE}

Música

Construccionismo

Irreversibilidades

Mediación

Vinculaciones

\section{KEYWORDS}

Music

Constructivism

Irreversibilities

Mediation

Attachments

\section{RESUMEN}

La obra de Antoine Hennion contiene un aporte específico y relevante para las ciencias sociales en general. Desde un campo de investigación que hasta cierto punto conforma una sub-disciplina los esfuerzos de Hennion intentan cuestionar los límites del construccionismo para dar lugar a una sociología de las irreversibilidades que integre en ensambles heterogéneos las relaciones entre lo humano y lo no humano. Ese campo, por ser el que más dificultad ofrece a esa maniobra, permite establecer el alcance de un giro que su obra acompaña y ayuda a fundar. La música ya no será sólo aquello que privilegia la musicología. Pero tampoco esto será negado en nombre de fuerzas sociales abstractas que parecen ser superiores a todas las realidades. La música será una relación, un arreglo heteróclito que debe ser aprehendido en esa complejidad. Desde esta posición nos ofrecerá la posibilidad de entender bajo nuevas luces tanto los objetos de la cultura como el papel clave de las decisiones y estrategias metodológicas en las investigaciones que su programa propone.

\section{ABSTRACT}

The work of Antoine Hennion contains a specific and relevant contribution to social sciences in general. From a field of research that forms a sub-discipline Hennion's efforts question the limits of constructivism to give rise to a sociology of irreversibilities that integrates the relations between the human and the non-human into heterogeneous arrangements. This field, which offers the greatest difficulty to this maneuver, establishes the scope of a turn that the work of Hennion accompanies and helps to found. Music will no longer be just what musicology privileges. But this will not be denied in the name of abstract social forces. Music will be a relationship, an heteroclite arrangement that must be apprehended within that complexity. From this position, and under a new light, Hennion will offer us the possibility to understand both the objects of culture and the key role of the decisions and methodological strategies in the research that his program proposes. 


\section{Acoger a los objetos, una propuesta llevada al límite}

Si el constructivismo en tanto consideración teórico-metodológica invita en cada ocasión a reanudar el análisis empírico para mostrar de qué manera la sociedad no se halla preconstituida, la transformación de esta apuesta en un axioma apriorístico y absoluto puede llevar a olvidar los efectos duraderos de las construcciones históricas, como si por ser fabricadas fueran evanescentes y reversibles. Los ritmos de la sociedad no coinciden con el impulso deconstruccionista de las ciencias sociales. Las transformaciones de largo plazo, las sedimentaciones de camadas de sentido, tienen efectivamente una duración y una eficacia que el antiesencialismo en piloto automático no puede aceptar: deconstruir cognitivamente no es anular los efectos de la historia y ni siquiera es conocer.

Pero esto que obliga a revisar al construccionismo como un lugar común a pesar de su fama de ser élmismo disolvente de las reificaciones no es suficiente. La profundización de este desafío es el punto al que nos llevan desde hace un tiempo obras como las de Antoine Hennion o Bruno Latour al plantear la doble necesidad de una sociología capaz de acoger los objetos (aquello que el construccionismo siempre logra tornar líquido en el juego de las resignificaciones constantes) y de asumir que la composición de lo social avanza a puntos de irreversibilidad que las dinámicas construccionistas, por definición, deben ignorar, pues para ellas la naturalización es el demonio.

Y por ello es preciso entender que darle positividad a un punto de vista superador del construccionismo, dejar de concebirlo como "post x" y asumir su propiedad implica tener en vista que el terreno en que recibimos una intervención como la de Hennion está marcado todavía por una herencia decimonónica en la que la división entre las ciencias naturales y las de la cultura, basada en una naturaleza universal y una cultura particularizante, ha sido puesta en cuestión en el mundo de las prácticas antes que en el ámbito reflexivo de las ciencias sociales: así todo nos condiciona para asumir contradictoriamente lo que esa intervención nos trae de novedad descomponiendo en purezas lo híbrido que es siempre lo realmente existente.

La obra de Hennion trabaja con las irreversibilidades para el caso de la música, enfrentándose al hecho de que en tanto realidad sonora es volátil y ofrece el máximo de resistencia a una sociología que se preocupe de ellas. Irreversibilidades,en tanto condicionamientos a los hechos futuros, consecuencias inevitables, a la vez ancladas en materia que se oponen como objeto a renovar los ciclos de la acción social, formando parte de ella misma. En su análisis de la disputa entre los barrocos y los modernos acerca de la manera adecuada de tocar la música antigua, previa a la constitución de la llamada "música clásica”, Hennion muestra una heterogeneidad de elementos que se pliegan unos a otros: unos instrumentos musicales diferentes a los actuales y "una manera de tocar, una sensibilidad, una posición o un gesto barrocos” (Hennion, 2002, p. 40) que definen el “famoso sonido" de otra época, traicionado por las reglas de la interpretación clásica. Frente a lo que un constructivista entendería como convenciones colectivas en las maneras de tocar y concebir la música, Hennion, por un lado, sospecha del poco énfasis que nuestro constructivista imaginario otorga a la controversia en estas convenciones y agrega a su vez lo que podría llamarse la "otra mitad” (Hennion, 2002, p. 361) implicada en la construcción del mundo: los dispositivos, los instrumentos, los montajes, en definitiva los objetos, comprendidos en su apertura, despliegue e inscripción en las tramas que hacen emerger la música. Elementos como "instrumentos, partituras, escenarios, medios de comunicación, intérpretes, profesores, productores, críticos...” (Hennion, 
2002, p. 25), son enumerados en distintos trabajos de Hennion para ilustrar de qué hablamos cuando hablamos de mediadores: estos no son conceptos ni abstracciones sino elementos concretos, activos, específicos para cada mediación musical. Esta clase de entramados humanos y no humanos realizan en cada ocasión un trabajo de mediación en la medida en que permiten, invitan, inhiben y/o detienen la constitución de distintos estados de la música. Si bien una lectura rápida de la obra de Hennion puede resaltar la fluidez y la permutación permanente entre estos estados, cada uno de ellos condiciona el regreso a un estado anterior y modela el estado siguiente.

La noción de mediación musical refiere a esta clase de entramados de humanos y objetos que produce irreversibilidades. El término no debe confundirse con el de intermediación, dado que no hablamos aquí de una actividad que pone en contacto, desplaza o difunde entidades preexistentes, sino de un trabajo que origina al mismo tiempo al objeto musical y a los sujetos del gusto que esa música exige. En particular, la idea de mediación no remite a los contenidos de ninguna teoría acerca de las mediatizaciones de la industria cultural, en tanto medios de producción, circulación y difusión de las obras musicales. Tampoco el concepto de mediación invoca un mero "rejunte" de elementos sino a una producción permanente, un trabajo concreto, en el que intervienen distintas agencias. Así las cosas, la música es el resultado de estas mediaciones, o más bien, la música es la propia relación de mediación y no tiene existencia por fuera de ella. El concepto de mediación, entonces, no resuelve nada de antemano: es una pregunta que se lanza cada vez a las músicas reales, particulares y concretas: ¿cómo están hechas?, ¿sobre qué arreglos se apoyan?, ¿qué hacen?, ¿qué hacen hacer?

En esa interrogación que define el corazón de la noción de mediación reside una apuesta por superar el dualismo: entre el sujeto y el objeto, entre la obra y la sociedad, entre lo humano y lo no humano, oposiciones que se sintetizan de acuerdo a Hennion en dos aproximaciones analíticas polares e igualmente reduccionistas del fenómeno musical: el esteticismo y el sociologismo. En un extremo, las lecturas estéticas de la música aceptan y comentan el objeto musical, al que consideran en tanto que obra autónoma, de la cual derivan los términos de la apreciación estética. Delimitan así los contornos de una "pareja trascendente" entre el objeto y el sujeto del gusto musical. En el otro polo, y en el intento de desligarse de la ponderación normativa de los esteticismos, como también del estatuto de excepción que otorgan al objeto y a la experiencia artística, las lecturas sociológicas disuelven la música en un juego social, ya sea de diferenciación o distinción entre los grupos, de lucha de clases o de identidad, prescindiendo de las características específicas del objeto musical, que se convierte en un mero signo arbitrario, y de los usos en los que se ve implicado. De esta manera, mientras en los esteticismos todo remite a la obra, en los sociologismos todo señala a la sociedad. En una propuesta de superación de estos dos polos de interpretación sobre la relación entre música y sociedad, Hennion recupera para el análisis las mediaciones, los “mixtos” de cosas y humanidad, conduciéndonos a un "repoblamiento del mundo musical” (Hennion, 2002, p. 19) en el que obra y sociedad, sujeto y objeto, se coproducen. La sociología de la mediación, en definitiva implica (discúlpese la extensión de la cita):

tomar en serio la inscripción de nuestras relaciones en las cosas, y no en deshacer con el pensamiento, como si no resistieran, los montajes y dispositivos a la vez físicos y sociales que sirven para establecer semejante reparto, situando de un lado un objeto autónomo y del otro un público sociologizable. Interpretar no es explicar, regresar a la 
pureza de las causas únicas, exteriores, que los actores buscan tanto como nosotros, sino mostrar las irreversibilidades que, por todas partes, han interpuesto los mixtos, entre los humanos, entre las cosas, entre los humanos y las cosas: ¿qué otra cosa es la música? (Hennion, 2002, p. 363).

El concepto de mediación y la idea de irreversibilidad pueden dilucidarse mejor en su contenido y alcance en diálogo con las consideraciones de Latour con las que la obra de Hennion guarda una relación de retroalimentación. Como plantea el propio Hennion, el concepto de mediación puede ser aplicado tanto a la música como a los proyectos técnicos (en efecto el concepto es usado por Latour -2012 - para explicar el trabajo de purificaciónen la constitución moderna de las ciencias y de la propia modernidad: purificar implica un esfuerzo de separación absoluta entre lo humano y lo no humano de lo que es, desde un inicio, híbrido). Pero para dar cuenta de la heterogeneidad y apertura de los objetos de la ciencia, Latour y Callon acuñaron por la misma época el concepto de traducción, noción adecuada a las especificidades de un objeto considerado cerrado e intocable por el consenso sociológico. Traducción, en tanto el proceso en el que convergen múltiples intereses que no sólo son transportados por humanos sino por objetos en el ensamblado de una red, que produce efectos en las relaciones y los cursos de acción (proceso que Latour abstrae de la lógica con que funciona la ciencia realmente existente, no la exaltada por el falsacionismo de gabinete ni la destituida por la epistemología crítica). Para el caso de la música, por el contrario, había que demostrar la resistencia de un objeto considerado evanescente, al punto de que todo en ella era “socialmente construido”. Dicho groseramente: una "sociología hospitalaria con los objetos”, como la llama Hennion en el artículo que presentamos aquí, es más difícil en el campo de la sociologíade la cultura que aparece reducida a signos, a espíritu, a "vapor” (por efecto de la purificación que Latour denuncia), que en el campo de la sociología de la ciencia en el que los razonamientos parecen más vinculados a la “materia”. Este argumento,que es ilustrativo y es sólo aceptable desde el punto de vista de la constitución moderna que Latour explica en sus fracasos (ya que cualquier historia del arte revela de inmediato hasta dónde, y en un sentido muy diferente al que afirmaban Marx y Engels, el sentido nace “tarado por la materia”), puede servirnos para precisar el valor heurístico de la noción de mediación.

Para el caso de la música, mediación era una noción más apropiadaal referir no sólo a los pasajes y asociaciones entre lo humano y lo no humano presentes en la idea de traducción, sino a la manera en que estas asociaciones habilitan o inhiben el juego social. Como lo expresa Hennion (2017,p. 5):

el mismo proyecto que, en el caso de la ciencia, consistía en hacer más social lo que era visto como objetivo, buscaba, al contrario, en el caso de la cultura, respetar la objetividad de lo que la sociología reducía a signos sociales, a marcas de diferenciación entre los grupos.

Es así que los conceptos de traducción y mediación se plantearon tareas diferentes y opuestas en un mismo programa de investigación. Cabe señalar que al mostrar la producción de irreversibilidades para el caso huidizo de la música, el concepto de mediación asumía un desafío mayor que el que asumía el de traducción en cuanto a la disputa con el sociologismo. De esta manera, podría pensarse que el concepto de mediación cumple más abarcativamente que el de traducción el programa global de dar cuenta de la agencia de los objetos que reúne a los dos autores. 


\section{Pragmatizar es complejizar}

La apertura al pragmatismo más recientemente desarrollada por Hennion profundiza, da nueva consistencia y un nuevo lenguaje a las adquisiciones consolidadas en La Pasión Musical. La apuesta por una forma de totalización que no implica ni cierre, ni unicidad, ni necesidad y el énfasis en la relación le dan positividad ontológica al movimiento que, al mismo tiempo, superaba el construccionismo y volvía a la sociología "hospitalaria con los objetos”. Esa apuesta y ese énfasis y su resultado pueden entenderse en un recorrido que comienza por "algo" que es al mismo tiempo básico y compuesto o complejo.

Ese "algo" es una consecuencia de que ni el sujeto es el centro del mundo ni las "cosas” están definitivamente hechas si no que ofrecen asideros "affordances" a los usos que esas mismas "cosas" sugieren y acaban aceptando en arreglos en que cosas y humanos participan. Una pelota, por ejemplo, no engendra las mismas prácticas lúdicas, ni se remite a la exclusiva posibilidad de habilitar juegos: puede ser adorno, mueble, objeto terapéutico.

Este régimen de determinación/indeterminación múltiple de los objetos respecto de los sujetos no puede concebirse con categorías como acción u objeto sino justamente a partir de las vinculaciones en que ellos existen en situaciones históricamente plurales y contingentes. Es allí donde una lectura del pragmatismo ofrece una solución si no confundimos pragmatismo con utilitarismo, la papelera de reciclaje a la que casi siempre se arroja la filosofía de James, Dewey y Peirce, entre otros. Tomado como una ontología, y no como un método, como una forma de referir al ser de lo existente y no como una forma de acceder a las cosas, el pragmatismo entiende que las cosas son las relaciones en que existen sujetos y cosas en usos y arreglos. Esas asociaciones son las pragmata en las que se integran indisolublemente aquello que el dualismo divide y opone.

Las pragmata existen como tales, como asociaciones en las que la agencia está distribuida en la asociación misma entre el sujeto y el mundo y no exclusivamente en el sujeto. Existen como posibilidad afirmada en un campo de posibilidades del que emergen como alternativa, superación, desvío, deriva, actualización no única y que define a ese campo como un pluriverso, una totalidad no cerrada y no sellada a la que referir las pragmata. En este sentido la pragmata abraza un conjunto: relaciones (primera complejidad) que solo pueden existir como parte de un conjunto de posibilidades de relación (segunda complejidad) que nos permiten entender, contra el sentido común, que el pragmatismo de esta concepción tiene poco de práctico si por esto se entiende simplificar, resolver e instrumentar.

Es en ese contexto que el análisis sociológico acompaña a las pragmata haciendo que emerjan como tales. Lo que obliga a un movimiento aparentemente contradictorio que redefine la noción de crítica a la cual se asocia la misión de la sociología. Cuando el análisis procede haciendo aparecer el mundo así lo que hace es

aceptarlo en su pluralidad, su apertura, la incertidumbre radical de los seres que surgen de él, sin nunca parar de cuestionar nuestras maneras de pensar. No hay necesidad de distancias críticas del afuera, el mundo es el mismo plural, abierto, en crisis permanente de tan crítico que es (Hennion, 2017, p. 13).

La tensión entre la aceptación y la crítica revela su carácter aparentemente contradictorio porque 
estos dos términos pierden su carácter opuesto cuando se considera la pluralidad del mundo y que la emergencia de un posible entre otros es al mismo tiempo aceptación de esa posibilidad/superación de cualquier noción de necesidad. Así, y al mismo tiempo, esto tiene una consecuencia específica para entender la posibilidad crítica que entraña el pragmatismo al que acude para especificarse una teoría de las mediaciones. Esta teoría que nace de la crítica al proyecto crítico, en tanto este instaura un punto de vista falsoy artificiosamente exterior para realizarse como tal, retorna a una de las propiedades que definían al proyecto crítico: este debe ser una crítica inmanente. Pues bien: despojado de la geometría que la dialéctica imponía al razonamiento de Adorno, y vuelto humilde por superación del filósofo héroe a favor del hombre común la tarea de aceptación del pragmatismo, que no puede ser sino crítica, porque cuestiona la fatalidad y la necesidad, se despliega desde el lugar mismo en que el mundo sucede, múltiple, o sea como inmanente. Yace aquí subentendida la devolución de la facultad crítica al "hombre común” que había emprendido Michel de Certeau, una influencia que pulsa como un bajo en la obra de Hennion. Y esto motiva la necesidad de sacar consecuencias metodológicas específicas del pragmatismo ontológico que da nuevo vigor a la idea de mediación.

\section{Otro sentido para la expresión etnografía}

Algunos etnógrafos, sobre todo algunos profesores de método etnográfico, acostumbran a decirnos, en un discurso escolarmente escandido, que el enfoque etnográfico, que no es ni el método ni el texto, implica una atención, un respeto por el punto de vista del nativo. Resta el pequeño problema de cuál es la teoría que permite identificar, interpretar y situar el "punto de vista del nativo" que de ninguna manera es evidente. La penumbra en la que permanece esa cuestión es el motivo por el cual la gran popularidad de la que goza el método etnográfico no necesariamente es acompañada de una conciencia crítica acercade lo problemáticas que resultan algunas de sus versiones, por ejemplo aquellas que acuden al punto de vista nativo para luego interpretarlo por una condición totalmente exterior al campo de interlocución en que se constituye una etnografía (es como resolver un juicio acudiendo a la jurisprudencia antes que al análisis de la singularidad del caso). Ha sido muy fácil en estos años leer etnografías en las que las artes interpretativas del sociólogo se basan antes que nada en saber aquello que el actor no sabe, en la posibilidad de inscribir el discurso nativo como portador de un sentido semipleno y contradictorio cuyo establecimiento y equilibrio definitivo depende del intérprete que propone un contexto no advertido por el sujeto para devolverle así alguna conciencia. Es preciso recordar aquí esas versiones y contradicciones por el punto de contraste que ofrecen al cruce fructífero que se da entre el método etnográfico y la intervención de Hennion con elementos presentes en este mismo.

De una forma que especifica la consideración pragmática, Hennion ha enfocado la investigación de los aficionados a diversas prácticas incluida la escucha musical. Ese conjunto de trabajos ayuda tanto a situar el problema que señalamos más arriba en la práctica del enfoque etnográfico y sus consecuencias en el plano metodológico, como a señalar una vía de superación a partir de la consideración del lugar clave que desempeñan operadores clave como lo son en el caso de la música o el consumo de vino, los "aficionados". No se puede seguir viendo en los aficionados la placa sensible que da cuenta de la exposición a los "rayos x" de la obra, las fuerzas ciegas del mercado y las inercias mudas de la estructura social condensadas en cada acto, sino un punto de anudamiento 
de una propensión que el aficionado cuestiona antes que nadie. Es necesario entender que en la actividad del aficionado no se despliega un "gusto" como actualización (simple o compleja) de una condición previa producto de las determinaciones de una habitualidad que se confronta a un estímulo, sino que es una actividad intensa, cooperativa, siempre en algún grado reflexiva, materialmente interpelada en la que lo que se establece es una vinculación, que es distinta de la expresión de una disposición previa en un conjunto de opciones que cumplen el papel del sucedáneo de la práctica. En una vinculación, el "gustador” trabaja para que el objeto lo llame y se haga amar por él en una situación de incertidumbres y cuestionamientos (nos volvemos fans, no nos revelamos tales y aunque creamos que el objeto nos "llama" como Dios a Pablo de Tarso, subyace a nuestra conversión descripta performáticamente como "repentina" un trabajo de engarzarse con las múltiples posibilidades del objeto y su agencia). La noción de "gusto adquirido", frecuente en el sentido común y que se usa para designar gustos difíciles pero socialmente "obligados”, como por ejemplo el mate o el alcohol, se pone en la pista de lo que la noción de vinculaciones pone en juego. Esta "rompe la oposición que acentúa el dualismo de la palabra gusto, entre una serie de causas que vendrían del exterior y el «hic et nunc» de la situación y de la interacción”(Hennion, 2010, p. página)y designa una tarea que no debe ser concebida como el efecto de declaraciones y etiquetas sino como algo que se relaciona con la actividad de las personas y con la capacidad de los objetos de "coproducir lo que pasa".

Así lo que se llama "sociología espontánea” de los actores es en realidad algo que surge de intentar desconocer que el aficionado, mucho antes que el sociólogo, pone en cuestión la determinación de sus gustos cuestionándolos por la "naturalidad” con que se imponen en su menú, por su carácter de moda, por la evidencia de la frontalidad con que el gusto es solicitado desde la oferta (como lo constata Hennion -2010-, los nativos también son bourdianos y suspicaces). En vez de una semi conciencia debe verse ahí una crítica en acto, un esfuerzo para hacer que un objeto se haga querer por ese sujeto que en ese movimiento prefigura el esfuerzo del sociólogo. Imaginando una sociología soñada y partiendo del papel clave que cumplen los aficionados, Hennion pregunta:

¿Y si, al contrario, escuchando a los aficionados, la sociología reconociera por fin este imperio, mejor dicho, el arte de una relación más intensa y reflexiva que, a través del gusto, los humanos establecen poco a poco con los objetos, con los demás, con su cuerpo y con ellos mismos?

Antes que repudiarlo, corregirlo o ponerlo en su lugar la sociología debe acompañar al aficionado todo el tiempo para dar cuenta de que con él aparece la pragmata. Esta surge en el campo etnográfico en tanto este es el campo de experiencia en que los esfuerzos, vaivenes y soluciones (en el sentido químico de la expresión) surgen en su verdad siempre particular. En ese sentido el aficionado es "profesor de pragmatismo" y su carácter de tal hace que no sea simplemente un agente (sea como efecto o como causa), un caso de una serie, sino el contrapunto de un diálogo que hace a la esencia de la sociología como ciencia histórica. 


\section{Notas}

1 En el año 2015, con el impulso y el apoyo de la Facultad de Humanidades y Ciencias de la Educación, Antoine Hennion visitó la Argentina para participar como profesor invitado del curso de posgrado “Sociología de los objetos estéticos y las mediaciones”, dictado por Pablo Semán y coordinado por Rodolfo Iuliano y Ornela Boix. Las líneas que siguen recogen algunas discusiones que tuvieron lugar entre los participantes del curso, como también en la conferencia impartida por Hennion, "Objetos estéticos, objetos sociológicos. De una sociología de la mediación a una pragmática de la vinculación”, base del artículo de su autoría que aquí publicamos.

\section{Referencias bibliográficas}

Hennion, A. (2002). La pasión musical. Barcelona: Paidós.

Hennion, A. (2010). Gustos musicales: de una sociología de la mediación a una pragmática del gusto. Comunicar, 17(34), 25-33.

Hennion, A. (2017). De una sociología de la mediación a una pragmática de las vinculaciones. Retrospectiva de un recorrido sociológico dentro del CSI. Cuestiones de sociología, 16, páginas.

Latour, B. (2012). Nunca fuimos modernos. Ensayo de antropología simétrica. Buenos Aires: Siglo XXI Editores. 